\title{
Atopische Erkrankungen Arzneimittelexantheme - stetige Zunahme in Industrieländern
}

\author{
A. Kreuter, P. Altmeyer, Dermatologische Klinik der Ruhr-Universität Bochum, Bochum \\ (Direktor: Prof. Dr. med. P. Altmeyer)
}

NOTFALLMEDIZIN 2003, 29: 242-243

Das Arzneimittelexanthem zeichnet sich durch eine nahezu unbegrenzte morphologische Vielfalt aus und zählt zu den häufigsten dermatologischen Krankheitsbildern. In zirka 5\% aller stationären Behandlungen führt eine Arzneimittelnebenwirkung zur Aufnahme. Der jährlich steigende Medikamentenkonsum in Deutschland, in Kombination mit den ständig neu auf den Markt kommenden Medikamenten der pharmazeutischen Industrie, erklären die sprunghafte Zunahme der Inzidenz von Arzneimittelexanthemen in den letzten Jahren.

K linisch können Arzneimittelexantheme wie infektiöse Exantheme - z. B. Röteln, Scharlach, Masern - erscheinen, aber auch makulopapulös, urtikariell, vesikulös oder hämorrhagisch imponieren. Rückschlüsse anhand der Morphe des Arzneimittelexanthems auf das auslösende Agens sind nicht möglich. Der weitaus häufigste Auslöser für Arzneimittelexantheme sind Penicilline und Sulfonamide (Tab. 1, Abb. 1).

Nicht selten kann durch eine sorgfältige Anamnese bei Verdacht auf eine Arzneimittelreaktion das

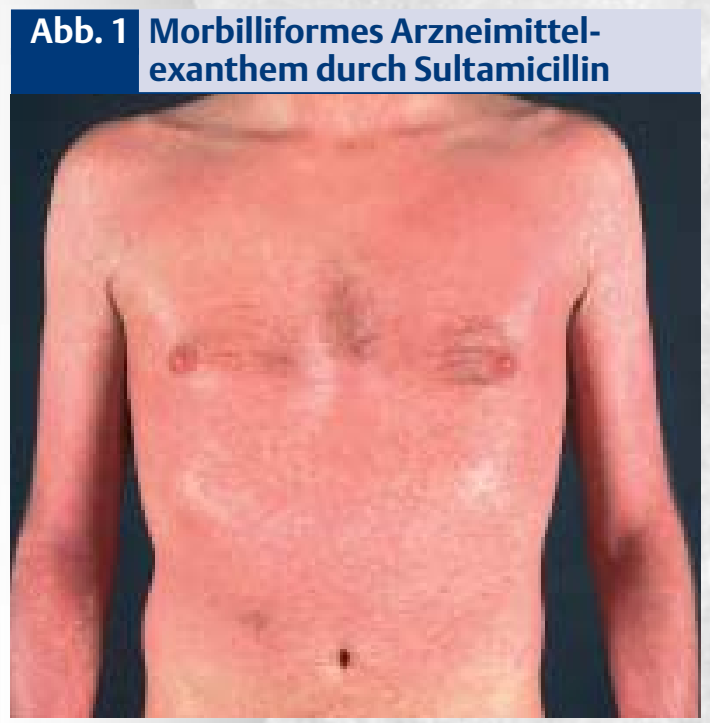

potentiell auslösende Medikament eruiert werden. Erfragt werden sollten zeitlicher Zusammenhang zur Medikamenteneinnahme, Anamnese bezüglich früherer Unverträglichkeitsreaktionen sowie bestehende internistische Grunderkrankungen. Erster Schritt in der Behandlung des Arzneimittelexanthems ist die Erkennung sowie das Absetzen der auslösenden Substanz. Bei schwerem Verlauf ist eine systemische Therapie mit Glukokortikosteroiden in Betracht zu ziehen, meist ist jedoch die Lokaltherapie mit Glukokortikosteroiden ausreichend. Bei starkem Pruritus können ergänzend Antihistaminika verabreicht werden. Auch wenn es nicht gelingt, das auslösende Medikament sicher zu identifizieren, sollte bei Verdacht auf eine entsprechende Substanz ein Warnpass ausgestellt werden.

\section{Urticaria}

Arzneimittel sind die häufigste Ursache für eine Urticaria. Es gibt kaum ein Medikament, das nicht eine akute Urticaria auslösen könnte. Bei der Urticaria handelt es sich um eine Sofortreaktion vom anaphylaktoiden Typ (Typ-I-Reaktion), bei der es meist schlagartig zu einem exanthematischen Quaddelschub am Integument kommt (Abb.

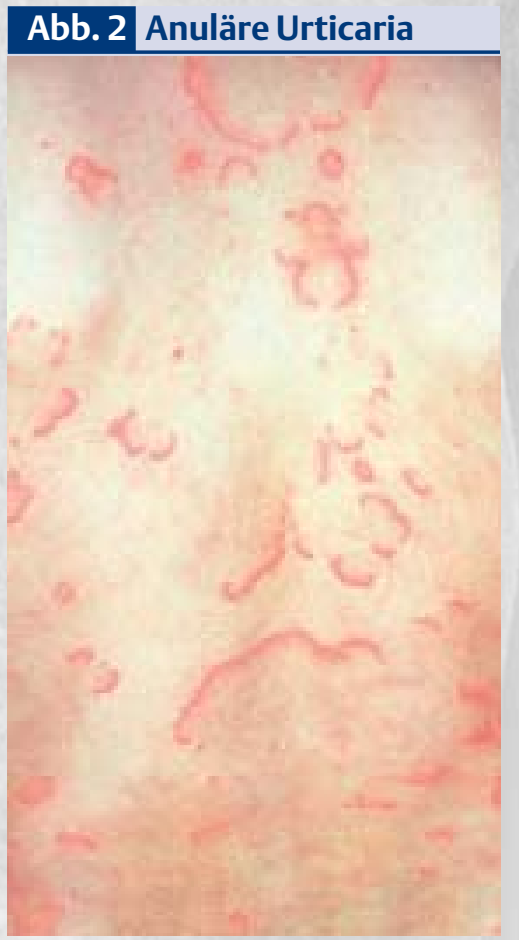

2). In der Mehrzahl der Fälle ist alleinig die Haut betroffen. Bei leichten Formen ist die Gabe eines Antihistaminikums ausreichend, bei generalisiertem Befall sollten kurzzeitig Glukokortikoide in hoher Dosierung (100-250 mg, gegebenenfalls bis $1000 \mathrm{mg}$ ) in Kombination mit H1Blockern (z.B. Fenistil ${ }^{\circledR}$ ) verabreicht werden. Begleitend sollte eine allergen- bzw. pseudoallergenarme Diät durchgeführt und gegebenenfalls bestehende akute Infektionsherde beseitigt werden. Bei Auftreten von Angioödem, Larynxödem, Glottisödem, Bronchospasmus sowie Kreislaufstörungen mit Schocksymptomatik ist eine sofortige intensivmedizinische Intervention mit Herz-Kreislauf-Kontrolle und Schocktherapie indiziert. 


\section{Erythema exsudativum multiforme}

Beim Erythema exsudativum multiforme (EEM) handelt es sich um eine akut auftretende Erkrankung mit charakteristischen kokardenförmigen, zur Konfluenz neigenden Effloreszenzen, die bevorzugt im Bereich der Extremitäten auftreten (Abb. 3). Die häufigsten Auslöser für einen EEM sind Herpes-simplexInfektionen sowie Arzneimittel. Bei der gefürchteten schwersten Verlaufsform eines EEM, dem StevensJohnson-Syndrom, kann es im Rahmen eines Schleimhautbefalls $\mathrm{zu}$ schmerzhaften Erosionen im Bereich des Mundes (Stomatitis) und der Augen (Konjunktivitis mit Gefahr narbiger Synechienbildung) sowie Befall der Genital- und Analschleimhaut kommen.

\section{Toxisch-epidermale \\ Nekrolyse (Lyell-Syndrom)}

Die schwerste Form einer Arzneimittelnebenwirkung an der Haut ist die toxisch-epidermale Nekrolyse (TEN, Lyell-Syndrom, Abb. 4). Die Letalität bei diesem Erkrankungsbild wird in der internationalen Literatur mit bis zu 30\% angegeben. Meist kommt es innerhalb kürzester Zeit nach Medikamenteneinnahme zu großflächigen Erythemen, subepidermaler Blasenbildung und letztendlich zur flächenhaften Nekrose der Epidermis. Patienten mit TEN werden wie Verbrennungspatienten unter intensiv-medizinischen Kautelen betreut. Neben engmaschiger Herz-Kreislauf-Kontrolle erfolgt eine Infektionsprophylaxe mit einem wenig allergenen Antibiotikum (z.B. Tetracycline) sowie Flüssigkeitsund Eiweißsubstitution nach Bilanzierung. Eine Therapie mit hochdo-

\section{Tab. 1 Häufige Auslöser von Arzneimittel- exanthemen}

- Sulfonamide

- Penicilline

- Cephalosporine

- Pyrazolone

- Salicylate

- Hydantoine

- Babiturate sierten systemischen Glukokortikosteroiden sollte sofort begonnen werden. Lokal werden antiseptische Folien (z.B. Metalline Folie ${ }^{\circledR}$ ) oder antiseptische Externa (z.B. Flammazine Creme $^{\circledR}$ ) verabreicht.

\section{Summary}

Drug-related exanthema is characterised by virtually unlimited morphological variety, and is one of the most common dermatological pathologies. Roughly $5 \%$ of all hospitalisations are due to the side effects of medication. The rapid increase in the incidence of drug-related exanthema may be explained by the annual increase in medication consumption in Germany, together with the constant stream of new drugs being brought onto the market by the pharmaceutical industry.

\section{Literatur}

1. Auquier-Dunant A, Mockenhaupt M, Naldi L. Correlations between clinical patterns and causes of erythema multiforme majus, Stevens-Johnson syndrome, and toxic epidermal necrolysis: results of an international prospective study. Arch Dermatol. 2002 ;138: 1019-1024

2. Babu KS, Belgi G. Management of cutaneous drug reactions. Curr Allergy Asthma Rep. 2002; 2: 26-33

3. Bachot N, Roujeau JC. Physiopathology and treatment of severe drug eruptions. Curr Opin Allergy Clin Immunol. 2001; 1: 293-298

4. Bigby M. Incidence, prevalence and the rates of cutaneous reactions to drugs. J Eur Acad Dermatol Venereol. 2000; 14: 435

5. Bigby M. Rates of cutaneous reactions to drugs. Arch Dermatol. 2001 Jun; 137: 765-770

6. Breathnach SM. Adverse cutaneous reactions to drugs. Clin Med. 2002; 2: 15-19 7. Del Rosso JQ. Skin manifestations of drug reactions. Curr Allergy Asthma Rep. 2002; 2: 282-287

8. Forman $\mathrm{R}$, Koren $\mathrm{G}$, Shear $\mathrm{NH}$. Erythema multiforme, Stevens-Johnson syndrome and toxic epidermal necrolysis in children: a review of 10 years' experience. Drug Saf. 2002; 25: 965-972

9. Fritsch PO, Sidoroff A. Drug-induced Stevens-Johnson syndrome/toxic epidermal necrolysis. Am J Clin Dermatol. 2000; 1 349-360

10. Ghislain PD, Roujeau JC. Treatment of severe drug reactions: Stevens-Johnson syndrome, toxic epidermal necrolysis and hypersensitivity syndrome. Dermatol Online J. 2002; 8: 5

11. Revuz J. New advances in severe adverse drug reactions. Dermatol Clin. 2001 Oct; 19: 697-709

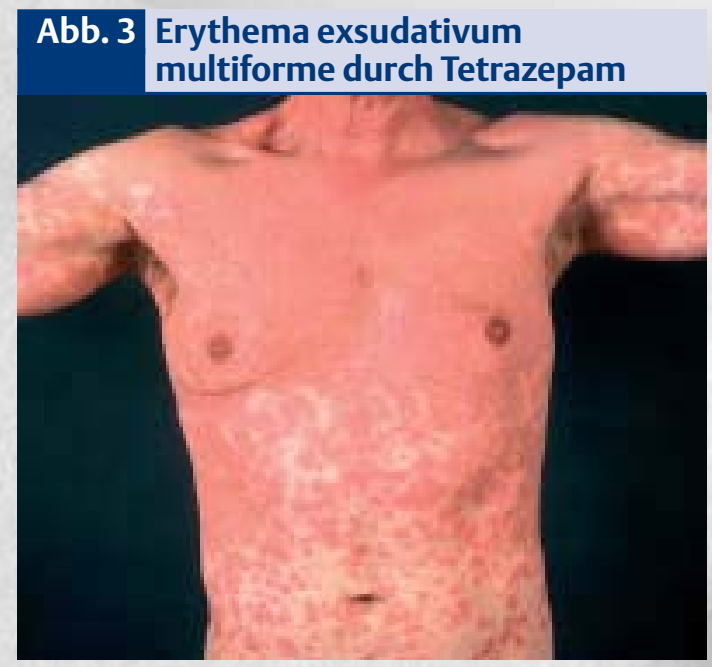

\section{Abb. 4 Toxisch epidermale Nekrolyse durch Metamizol}

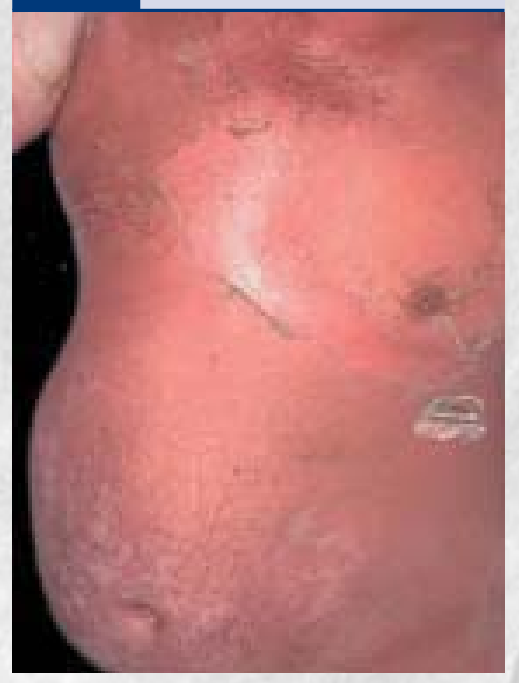

12. Sullivan JR, Shear NH. Drug eruptions and other adverse drug effects in aged skin. Clin Geriatr Med. 2002; 18: 21-42

13. Svensson CK, Cowen EW, Gaspari AA. Cutaneous drug reactions. Pharmacol Rev. 2001; 53: 357-379

14. Ward HA, Russo GG, Shrum J. Cutaneous manifestations of antiretroviral therapy. J Am Acad Dermatol. 2002; 46: 284-293

15. Warnock JK, Morris DW. Adverse cutaneous reactions to antipsychotics. Am J Clin Dermatol. 2002; 3: 629-636

\section{Anschrift für die Verfasser}

Dr. med. Alexander Kreuter Dermatologische Klinik der

Ruhr-Universität Bochum

Gudrunstraße 56

44791 Bochum 